\title{
Possibilities of microemulsion application in rectal administration of indomethacin
}

Special Issue Article

\author{
${ }^{1}$ Comenius University, Faculty of Pharmacy, \\ Department of Galenic Pharmacy, Odbojárov \\ 10 SK-832 32, Bratislava, Slovakia \\ ${ }^{2}$ Slovak Academy of Sciences, Centre of Experimental \\ Medicine, Institute of Experimental Pharmacology and \\ Toxicology, Dúbravská cesta 9 \\ SK-841 04 Bratislava, Slovakia
}

Špaglová M. ${ }^{1}$, Čuchorová M. ${ }^{1}$, Čierna M. ${ }^{1}$, Mikušová V. ${ }^{1}$, Bauerová K. ${ }^{2}$, Poništ S. ${ }^{2}$

Received 14 June, 2021, accepted 27 July, 2021

Abstract Rectal administration is a suitable route of administration for drugs that are either very irritating to the intestine (e.g., indomethacin) or are more effective when the first-pass effect in the liver is circumvented. Microemulsions are a tool for the improvement of penetration of sparingly soluble drugs. They are mainly used in topical and transdermal drug delivery. However they find application also in other routes of administration, mainly due to their ability to solubilize sparingly soluble drugs. The selection of a suppository base depends on the physical properties of the drug. The study focused on evaluating the effect of the microemulsion as the solubilizer of sparingly soluble indomethacin in hydrophilic and lipophilic suppository bases compared with Polysorbate 80 as the excipient contained in the microemulsion. The reference suppositories were prepared by the traditional moulding technique from Adeps solidus or Macrogol suppository base without the previous drug solubilization. The microemulsion-based suppositories were prepared after the initial solubilization of the drug in the microemulsion or Polysorbate 80 , followed by the addition of suppository base to maintain the same drug/solubilizer ratio. The suppositories were tested for softening time, hardness, and uniformity of mass. The dissolution test was performed using the dialysis tubing method in the basket apparatus. The amount of indomethacin released into the dissolution medium was determined spectrophotometrically at $320 \mathrm{~nm}$. The results indicate that solubilization of indomethacin in the microemulsion had a positive effect on in vitro drug release but not as significant as in the case of Polysorbate 80 used alone. The enhancement ratio for Polysorbate 80 in Adeps suppositories was 2.9, for the microemulsion in Adeps suppositories was 1.1, and for Polysorbate 80 in Macrogol suppositories was 7.4 after 3 hours. The test of uniformity of mass had shown that all suppositories (reference, solubilizer-containing) are within the permitted limits. The softening time was reduced by adding the solubilizer to each type of suppository base.

Keywords microemulsion - indomethacin-suppository-Polysorbate 80 -macrogol-Adeps solidus

\section{INTRODUCTION}

In practice, it is not always possible to administer drugs orally, because of patients' nausea, vomiting, or drug properties causing gastric irritation, for example, or when it is inconvenient, as with infants and elderly people. Rectal administration represents an alternative for systemic and local drug delivery. The advantages of rectal administration are an environment without enzymatic or microbial activity (bacteroids, fecal bacterium) acting on the drug compared with other parts of the gastrointestinal tract. In rectal drug application, the first-pass effect is avoided as well as a potential drug metabolism to inactive metabolites (Blumer, 2011; Hua, 2019). The drug delivery from the colorectal lumen to the rectal mucosa could be limited by its ability to dissolve and diffuse through the mucus. This is the main difficulty especially for large and sparingly soluble drugs (Hua, 2019). To increase the bioavailability of drugs from suppositories, it is important to select a suitable suppository base. The application of drug nanocarriers is possible also in rectal administration. The studies focused on rectal drug delivery have evaluated nanoparticles, polymer micelles, liposomes, nanoemulsions, etc. The beneficial effect of the nanoemulsion has been observed, for example, for the release of indomethacin or diazepam. Despite interesting results, they have not received much attention in recent 
years (Melo et al., 2018). Self-microemulsifying suppositories containing an oil phase, a surfactant, a co-surfactant, and a drug that forms o/w microemulsion after gentle agitation in aqueous media can also be promising in rectal administration (Gugulothu et al., 2010).

\section{METHODS}

Indomethacin (IND) was purchased from Valúch S.R.O. (Banská Bystrica, Slovakia). Macrogol 4,000, Macrogol 6,000, animal fat (Adeps solidus), Polysorbate 80, isopropyl alcohol, oleic acid, and ethanol (96\%) were obtained from Centralchem S.R.O. (Bratislava, Slovakia). Isopropyl myristate was purchased from Sigma-Aldrich Chemie GmbH (Steinheim, Germany). Purified water was prepared with a distillation apparatus (Kavalier, Czech Republic).

\section{Preparation of indomethacin suppositories}

The Macrogol suppository base was prepared by melting and homogening Macrogol 4,000 (33\%; w/w), Macrogol 6,000 (47 \%; w/w) and purified water (20\%; w/w). The suppository base was completely melted. Indomethacin was either directly suspended in the melted suppository base (Adeps solidus or Macrogol mixture) and filled into the mould cavities (reference suppositories) or the drug was at first solubilized in Polysorbate 80 or the microemulsion (ME) consisting of purified water (31\%; w/w), isopropyl myristate (16\%; w/w), isopropyl alcohol (26.5\%; w/w) and Polysorbate 80 (26.5\%; $\mathrm{w} / \mathrm{w}$ ) so that the final drug to solubilizer ratio was 1 to 5.7 . The required amount of the suppository base (+/- solubilizer) was determined experimentally. The composition of the suppositories is shown in Table 1.

\section{Quality tests of suppositories}

a) Hardness test: 10 suppositories of each type (the reference, the solubilizer-containing Macrogol or Adeps) were randomly selected, and their hardness was tested using a hardness tester (Schleuniger 2E, Germany)

b) Uniformity of mass: 20 suppositories of each type were weighed individually. It was to find out whether they are within the allowed limits.

c) Softening time was determined according to the European Pharmacopoeia 10.0 (2.9.22 Softening time determination of lipophilic suppositories) with a single modification; $20 \mathrm{ml}$ of purified water, instead of $10 \mathrm{ml}$, was used to cover the entire volume of the suppository. Three parallel measurements were carried out.

\section{In vitro drug release study}

The basket type of dissolution tester (Erweka DT 6, Germany) was used to evaluate the drug release rate from suppositories. As the dissolution medium, $900 \mathrm{ml}$ of purified water was used which was constantly heated to $37 \pm 0.5^{\circ} \mathrm{C}$. The suppository was inserted into the dialysis membrane pocket (Spectra/ Por 4, Spectrum Laboratories, California, USA) and then placed into the metal basket rotating at $50 \mathrm{rpm}$. At specific time intervals $(5,10,15,30,60,120$, and 180 minutes), a volume of $4 \mathrm{ml}$ of dissolution medium was withdrawn to analyze drug concentration. The drug content in the samples was determined spectrophotometrically (Thermo Scientific Genesys 10S, USA) at wavelength $320 \mathrm{~nm}$ against the dissolution medium using slope and intercept from linear regression of the standard curve to express the drug concentration in the samples. The values obtained from dissolution test with placebo were taken into account.

\section{Statistical analysis}

Data are expressed as the mean \pm S.D. Statistical significance was analyzed using Student's $t$-test for the comparison of two groups. Differences at $p<0.05$ were considered statistically significant. The statistical analysis was performed using Microsoft Office Excel 2016 with the add-in Daniel's XL Toolbox.

\section{RESULTS}

Table 1. The composition of indomethacin suppositories (the mass for preparation of 14 supp.).

\begin{tabular}{|c|c|c|c|c|c|c|}
\hline & $\begin{array}{c}\text { Supp. } \\
1\end{array}$ & $\begin{array}{c}\text { Supp. } \\
2\end{array}$ & $\begin{array}{c}\text { Supp. } \\
3\end{array}$ & $\begin{array}{c}\text { Supp. } \\
4\end{array}$ & $\begin{array}{c}\text { Supp. } \\
5\end{array}$ & $\begin{array}{c}\text { Supp. } \\
6\end{array}$ \\
\hline $\begin{array}{c}\text { Indomethacin } \\
\text { (g) }\end{array}$ & 1.4 & 1.4 & 1.4 & 1.4 & 1.4 & 1.4 \\
\hline $\begin{array}{c}\text { Polysorbate } \\
80(\mathrm{~g})\end{array}$ & - & 7.9 & - & - & 7.9 & - \\
\hline $\begin{array}{c}\text { Microemulsion } \\
\text { (g) }\end{array}$ & - & - & 7.9 & - & - & 7.9 \\
\hline $\begin{array}{c}\text { Adeps base } \\
\text { (g) }\end{array}$ & 28.0 & 18.7 & 18.7 & - & - & - \\
\hline $\begin{array}{c}\text { Macrogol base } \\
\text { (g) }\end{array}$ & - & - & - & 33.1 & 23.9 & 23.9 \\
\hline
\end{tabular}

Table 2. The comparison of indomethacin suppositories through physical parameters.

\begin{tabular}{|c|c|c|c|}
\hline & $\begin{array}{c}\text { Uniformity } \\
\text { of mass }(\mathrm{g})\end{array}$ & $\begin{array}{c}\text { Hardness } \\
(\mathrm{N})\end{array}$ & $\begin{array}{c}\text { Softening } \\
\text { time }(\mathrm{s})\end{array}$ \\
\hline Adeps & $1.97 \pm 0.01$ & $76.3 \pm 18,6$ & $474.0 \pm 8.5$ \\
\hline $\begin{array}{c}\text { Adeps + } \\
\text { Polysorbate } 80\end{array}$ & $2.06 \pm 0.02$ & $54.3 \pm 4.5$ & $361.0 \pm 80.6$ \\
\hline Adeps + ME & $1.93 \pm 0.03$ & $51.6 \pm 10.6$ & $261.0 \pm 42.4$ \\
\hline Macrogol & $2.33 \pm 0.07$ & - & $2265.0 \pm 190.9$ \\
\hline $\begin{array}{c}\text { Macrogol + } \\
\text { Polysorbate } 80\end{array}$ & $2.36 \pm 0.02$ & - & $1680.0 \pm 84.9$ \\
\hline
\end{tabular}

(-) not possible to evaluate, suppositories did not break but deformed 


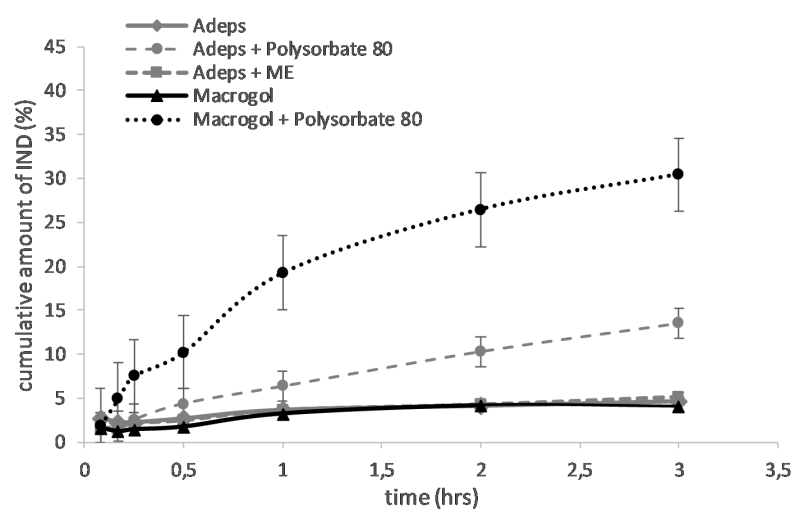

Figure 1. The dissolution profiles of indomethacin from different suppository bases depending on the presence of the solubilizer (Polysorbate 80/ME).

\section{DISCUSSION}

Suppositories containing the nonsteroidal anti-inflammatory drug indomethacin are used mainly to alleviate of pain and treat inflammation, arthritis caused by gout, osteoarthritis, etc. Indomethacin suppositories from two manufacturers are currently registered in Slovakia: one formulation contains only hard fat and the drug, and the second the addition of maize starch other than hard fat and drug (adc.sk, 2020). The suppositories have to be of optimal consistency, hard enough for trouble-free manual insertion into the rectum, and at the same time sufficiently resistant to breakage and melting under storage conditions. They must not start melting during insertion into the body cavity; therefore, their melting point must not be lower than $20^{\circ} \mathrm{C}$. At the same time, no sedimentation of the drug can occur during storage. The suppositories have to melt in the rectum as soon as possible after application. This also prevents the unpleasant feeling of pressure of the foreign body in the rectum and subsequently leads to a rapid release of the drug, ensuring a rapid local or systemic effect of the drug (Hua, 2019).

The sixth formulation of suppositories was not successfully prepared. It appears that the presence of the microemulsion in the Macrogol base caused a significant decrease in the melting point, and they were therefore unable to solidify. The test of uniformity of mass was performed by weighing 20 individual suppositories (reference, i.e., indomethacin suppositories without the solubilizer and indomethacin suppositories prepared after the drug solubilization in $\mathrm{ME}$ or Polysorbate 80 ). The average mass of the suppositories and the standard deviation are recorded in Table 2. All suppositories prepared were in the range of acceptable values (with a tolerance of up to $\pm 5 \%$ and maximally up to $\pm 10 \%$ in two cases). The softening time of the suppositories decreased due to the presence of Polysorbate 80/ME in Adeps base from 474 seconds to $361 / 261$ seconds (i.e., from approximately 7.9 minutes to $6.0 / 4.4$ minutes). Macrogol suppositories were much more resistant with softening time of 37 minutes. The drug solubilization in Polysorbate 80 caused a decrease in softening time to 28 minutes. The suppositories appeared to be stable at the storage temperature of $4^{\circ} \mathrm{C}$. After 30 days of storage they did not show visible signs of deterioration.

The drug solubilization in Polysorbate 80 caused a significant increase in drug release from both suppository bases ( $p$ $<0.05$ ). As Figure 1 indicates, indomethacin diffused to a greater extent across the dialysis membrane from a Macrogol suppository base with Polysorbate 80 . However, the quality is on side of the suppositories from Adeps solidus. Macrogol suppositories with Polysorbate 80 tended to deform at the tip, which could cause difficulty during insertion into the rectum. Perhaps, for this reason, Adeps solidus is used for the commercial production of indomethacin suppositories, although the rule that hydrophilic suppository bases should be used for the processing of sparingly water-soluble drugs is not maintained.

Unlike Polysorbate 80 , the polysorbate-containing microemulsion increased the cumulative amount of diffused drug from Adeps solidus only slightly (+10\%). Since it was an o/w type of microemulsion, it could be assumed that indomethacin is better solubilized in the oil droplets of the microemulsion and is difficult to remove from these droplets. To quantify the effectiveness of the drug solubilizer in the suppositories, an enhancement ratio (ER) was calculated. It is the ratio of the amount of the drug released (\%) using a solubilizer to the amount of drug released (\%) from the reference sample. ER for Polysorbate 80 in Adeps suppositories was 2.9, for the ME in Adeps suppositories it was 1.1, and for Polysorbate 80 in the Macrogol suppositories it was 7.4 after 3 hours.

The beneficial effect of Polysorbate on drug release from suppositories has been described in several studies (Hanaee et al., 2004; Constantin, 2013; Alsamman and Othman, 2017; Szulc-Musiol et al., 2020). The reasons may be various, e.g., an increase of the exposed surface of the suppository mass in the rectum, a decrease of the interfacial tension between the base and the rectal fluid, or better wetting of the drug (Hanaee et al., 2004). Sah et al. (Sah and Saini, 2008) observed the effect of Polysorbate 80 on the release of indomethacin but at significantly lower concentrations (1\% / 5\% w / w). Drug concentration increased only when a low concentration of Polysorbate 80 was used (1\%, w / w). The authors have mentioned a similar opinion that at higher concentrations Polysorbate 80 forms micelles from which the drug cannot easily escape.

\section{CONCLUSION}

Due to the content of an oil phase and a mixture of surfactants, even compound systems such as microemulsions and nanoemulsions can be considered as potentially synergistically acting drug absorption enhancers. Their application in rectal administration is still limited. The dissolution profiles of indomethacin from suppository 
bases do not indicate a significant improvement after drug solubilization in the microemulsion. The drug is probably well solubilized in the oil phase of the microemulsion and is therefore released more slowly than when Polysorbate 80 is used alone. Whether the microemulsion tends to prolong drug release similar to some self-microemulsifying suppositories could be evaluated if the dissolution test time were prolonged. As previous studies have confirmed, Polysorbate 80 is an effective drug solubilizer in suppositories, and on the basis of these results it could be concluded that its influence is higher in the hydrophilic suppository base. Thus, the synergistic effect of the absorption enhancers contained in the microemulsion has not been confirmed.

\section{ACKNOWLEDGEMENTS}

The study was supported by a VEGA 2/0115/19 grant.

\section{References}

[1] ADC.sk. Indomethacin. Available at https://www.adc. sk/databazy/produkty/detail/indometacin-100-berlinchemie-439509.html (Accessed 14 June 2021).

[2] Alsamman A, Othman M. Preparation and in vitro evaluation of fast release diazepam suppositories for febrile seizures. Asian $J$ Pharm Clin Res. 2017;10:224-230.

[3] Blumer JL. Principles of drug disposition in the critically ill child. In Fuhrman BP \& Zimmerman JJ. Pediatric Critical Care: Saint Louis: Mosby; 2011.

[4] Constantin 10. Development of misoprostol suppositories for postpartum hemorrhage. Pharmacol Pharm. 2013;4:71-76.

[5] Council of Europe. European pharmacopoeia 10.0: Softening time determination of lipophilic suppositories (chapter 2.9.22). Strassbourg: EDQM; 2019.

[6] Gugulothu D, Pathak S, Suryavanshi S, Sharma S, PatravaleV. Selfmicroemulsifiyng suppository formulation of $\beta$-artemether. AAPS PharmSciTech. 2010;11:1,179-1,184.
[7] Hanaee J, Javadzadeh Y, Taftachi S, Farid D, Nokhodchi A. The role of various surfactants on the release of salbutamol from suppositories. Farmaco. 2004; 59: 903-906.

[8] Hua S. Physiological and pharmaceutical considerations for rectal drug formulations. Front Pharmacol. 2019;10:1,196.

[9] Melo M, Nunes R, Sarmento B, Neves J. Rectal administration of nanosystems: From drug delivery to diagnostics. Mater Today Chem. 2018;10:128-141.

[10] Sah M, Saini T. Formulation Development and Release Studies of Indomethacin Suppositories. Indian J Pharm Sci. 2008;70:498501

[11] Szulc-Musiol B, Bulas L, Dolinska B. Effect of Selected Surfactants on Kinetics of Meloxicam Release from Rectal Suppositories. Indian J Pharm Sci. 2019;81:1,115-1,121. 